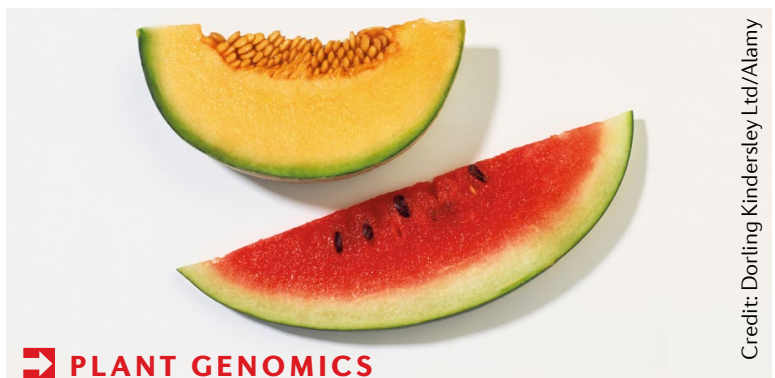

\section{Sequencing bears fruit}

Strategies for crop improvements require an in-depth understanding of plant domestication history. Two studies in Nature Genetics provide high-resolution maps of genetic variation across melon and watermelon species, respectively, and identify candidate loci linked to fruit quality traits through genome-wide association studies (GWAS) and population genomic analyses.

Zhao et al. re-sequenced the genomes of 1,175 diverse accessions of melon (Cucumis melo L.), including 134 wild and 1,041 cultivated accessions, to characterize the fruit's population structure and domestication history, which hitherto had been inferred predominantly from molecular marker analyses as well as archaeological and historical data. A phylogenetic tree, built on the basis of a subset of 17,055 single-nucleotide polymorphisms (SNPs) at fourfold degenerate sites, indicated the existence of three distinct clades with separate botanical characteristics. The authors speculate that three independent domestication events occurred, one in Africa and two in India. Focusing on the clades from India, the team identified independent domestication events for the two major melon subspecies, C. melo ssp. melo and C. melo ssp. agrestis, with distinct domestication mechanisms for fruit mass, flesh bitterness and acidity. A GWAS for 16 agronomic traits (for example, flesh and peel colour) using 1,067 diverse accessions identified 208 significant association signals in the melon genome.

Guo et al. focused on another Cucurbitaceae family member, the watermelon (Citrullus lanatus), which was known to originate in Africa. First, the authors improved the watermelon cultivar '97103' reference genome, assembling a de novo genome from PacBio long reads, BioNano optical maps and $\mathrm{Hi}-\mathrm{C}$ chromatin interaction maps. Next, they re-sequenced 414 Citrullus accessions, representing all 7 extant species and various geographical locations, and inferred phylogenetic relationships using 89,914 SNPs at fourfold degenerate sites. A GWAS for 7 fruit quality traits, including sugar content and fruit shape, identified 43 association signals, of which 8 overlapped previously identified loci.

These studies provide useful genomic resources for further analysis. Knowledge of the genes linked to domestication and agronomic traits will be valuable for breeding these important fruit crops.

Linda Koch

ORIGINAL ARTICLES Zhao, G. et al. A comprehensive genome variation map of melon identifies multiple domestication events and loci influencing agronomic traits. Nat. Genet. 51, 1607-1615 (2019) | Guo, S. et al. Resequencing of 414 cultivated and wild watermelon accessions identifies selection for fruit quality traits. Nat. Genet. $\mathbf{5 1}$ 1616-1623 (2019)

RELATED ARTICLE Klee, H. J. \& Tieman, D. M. The genetics of fruit flavour preferences. Nat. Rev. Genet. 19, 347-356 (2018)

\title{
Insights from Ugandan genomes
}

Studies assessing genetic diversity in global populations are important to understand the impact of genetic variation on disease risk, but limited numbers of genomewide association studies (GWAS) have been performed in nonEuropean populations. Genetic variation is higher in African populations than in European populations, which suggests more opportunities for discovery per individual in populations with African ancestry. A new paper in Cell provides the largest wholegenome sequence data set in an African population to date and shows that genetic causes of disease can differ between African and European populations.

Gurdasani et al. collected and analysed genome-wide data from approximately 6,400 individuals in a rural Ugandan community, obtaining whole-genome sequences from nearly 2,000 of them. In addition, data from nearly 8,000 individuals from Ghana, Kenya, Nigeria and South Africa were analysed. An electronic questionnaire was used to collect information on demographics and non-communicable diseases, and physical attributes, such as height, weight and blood pressure, and serum levels of lipids and haemoglobin $\mathrm{A}_{1 \mathrm{c}}\left(\mathrm{HbA}_{1 \mathrm{c}}\right)$ were measured.

Ugandan genomes were a mixture of many ancestries, including ancient East African populations and Eurasian genomes, indicating a complex history of migration into the region. As expected, the authors found that Africans had higher levels of genetic variation than other continental populations.

In a GWAS of 34 cardiometabolic traits in up to $\sim 14,000$ individuals from the African populations, the researchers identified new genetic associations between variants unique to Africans (or rare in non-Africans) and blood, liver function and glucose-related traits. For example, a novel association was identified between a deletion known to cause a-thalassaemia in African individuals and $\mathrm{HbA}_{1 \mathrm{c}}$ levels. The deletion is present in $22 \%$ of Africans and protective against severe malaria. As $\mathrm{HbA}_{1 \mathrm{c}}$ levels are often used to diagnose diabetes mellitus, the authors say its utility as a diagnostic test in African populations needs to be evaluated.

Height was less genetically determined in the rural Ugandan population than in Europeans, perhaps due to nutritional deficits that attenuate the effects of genetic variance on height. By contrast, LDL cholesterol levels were more genetically determined in Ugandans than in Europeans, potentially as a result of increased levels of obesity that reduce the influence of genetic factors on LDL cholesterol levels.

"This study confirms that genetic causes of disease may be different in Africans and provides opportunities to identify new genes associated with disease that would not be identified in European studies," says Gurdasani. "This kind of research will allow us to identify new targets for therapies that could potentially be useful for all populations."

Rebecca Kelsey

ORIGINAL ARTICLE Gurdasani, D. et al. Uganda genome resource enables insights into population history and genomic discovery in Africa. Cell 179 , 984-1002 (2019)

RELATED ARTICLE Gurdasani, D. et al. Genomics of disease risk in globally diverse populations. Nat. Rev. Genet. 20, 520-535 (2019)

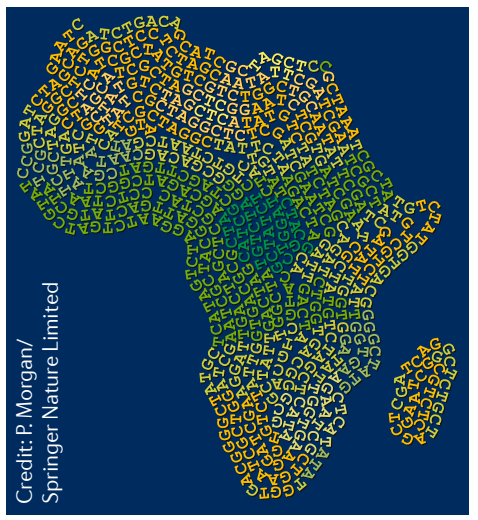

